ANNALES

POLONICI MATHEMATICI

$83.3(2004)$

\title{
Matrix inequalities and the complex Monge-Ampère operator
}

\author{
by JONAS WIKLUND (Umeå)
}

\begin{abstract}
We study two known theorems regarding Hermitian matrices: Bellman's principle and Hadamard's theorem. Then we apply them to problems for the complex Monge-Ampère operator. We use Bellman's principle and the theory for plurisubharmonic functions of finite energy to prove a version of subadditivity for the complex MongeAmpère operator. Then we show how Hadamard's theorem can be extended to polyradial plurisubharmonic functions.
\end{abstract}

1. Introduction. We begin our study with a matrix equality that is reasonably well known in pluripotential theory, nevertheless the proof is not as simple as it may appear, so we give a careful proof of all steps.

Lemma 1.1 (Bellman's principle [Gav77]). Let $\mathcal{A}$ denote the family of all positive definite $n \times n$ Hermitian matrices with determinant 1 . For any positive definite Hermitian matrix $B$ we have

$$
(\operatorname{det} B)^{1 / n}=\frac{1}{n} \inf _{A \in \mathcal{A}} \operatorname{tr}(A B) .
$$

To prove the equality in Lemma 1.1 one first shows that for an arbitrary matrix $A \in \mathcal{A}$ the left-hand side is less than the right-hand side, which would surely be a simple matter if the product of two symmetric matrices were symmetric. Since it is symmetric only if the matrices commute, some preliminary work is necessary.

The proof of the lemma above is based on conjunctive reduction of two matrices. The principle of conjunctive reduction can be found in the literature (see e.g. [MM64].) Furthermore a careful proof of Lemma 1.1 gives explicit algorithms for calculating the matrix $A$ for a given matrix $B$.

2000 Mathematics Subject Classification: 32F07, 32U25.

Key words and phrases: complex Monge-Ampère operator, plurisubharmonic functions, Lelong numbers. 
Theorem 1.2. Let $A$ and $B$ be two Hermitian matrices, and let $A$ be positive definite. Then there is a nonsingular matrix $Q$ such that

$$
\begin{aligned}
& Q^{*} A Q=I, \\
& Q^{*} B Q=\operatorname{diag}\left(k_{1}, \ldots, k_{n}\right)=D_{k},
\end{aligned}
$$

where $D_{k}$ is the diagonal matrix with the eigenvalues of $A^{-1} B$ as entries.

Proof. Note that $A^{1 / 2}$ and $A^{-1 / 2}$ are both positive semidefinite Hermitian matrices. Thus $A^{-1 / 2} B A^{-1 / 2}$ is Hermitian. Since

$$
\begin{aligned}
& \operatorname{det}\left(A^{-1} B-\lambda I\right) \\
& \quad=\operatorname{det}\left(A^{1 / 2}\right) \operatorname{det}\left(A^{-1} B-\lambda I\right) \operatorname{det}\left(A^{-1 / 2}\right)=\operatorname{det}\left(A^{1 / 2}\left(A^{-1} B-\lambda I\right) A^{-1 / 2}\right) \\
& \quad=\operatorname{det}\left(A^{-1 / 2} B A^{-1 / 2}-\lambda I\right),
\end{aligned}
$$

it follows that $A^{-1} B$ has real eigenvalues $\left(k_{1}, \ldots, k_{n}\right)$. Let $P$ be a unitary matrix such that $P^{*} A^{-1 / 2} B A^{-1 / 2} P=\operatorname{diag}\left(k_{1}, \ldots, k_{n}\right)$. Finally, let $Q=$ $A^{-1 / 2} P$.

1.1. Proof of Lemma 1.1. Let $A$ and $B$ be as in Theorem 1.2, with $\operatorname{det} A=1$. With the above notation,

$$
\begin{aligned}
\operatorname{det} B & =\operatorname{det}\left(A^{-1} B\right)=\prod k_{i} \\
& \leq\left(\frac{1}{n} \sum k_{i}\right)^{n}=\left(\frac{1}{n} \operatorname{tr}\left(D_{k}\right)\right)^{n} \\
& =\left(\frac{1}{n} \operatorname{tr}\left(Q^{*} B Q\right)\right)^{n}=\left(\frac{1}{n} \operatorname{tr}\left(Q Q^{*} B\right)\right)^{n}=\left(\frac{1}{n} \operatorname{tr}\left(A^{-1} B\right)\right)^{n},
\end{aligned}
$$

where the second to last equality follows from the fact that $\operatorname{tr}(F G)=\operatorname{tr}(G F)$ for any two matrices $F$ and $G$. The last equality follows from the definition of $Q$.

To show that the infimum is attained, suppose $B$ is diagonal. If $\operatorname{det} B \neq 0$, we take $A$ as the diagonal matrix with diagonal elements $a_{i i}=b_{i i}^{-1}(\operatorname{det} B)^{1 / n}$. On the other hand, if $\operatorname{det} B=0$, then - since $B$ is diagonal — we have $b_{i i}=0$ if $i \in \Lambda$, for some index set $\Lambda \subset\{1, \ldots, n\}$. Let $k$ be the number of elements in $\Lambda$. Take $A$ to be a diagonal matrix. With this notation we have

$$
\operatorname{tr}(A B)=\sum_{i \notin \Lambda} a_{i i} b_{i i}
$$

So if we choose $a_{i i}=1 / k$ if $i \in \Lambda$, we have $\operatorname{tr}(A B)=k^{-1} \operatorname{tr}(B)$. Take $a_{i i}=k^{(n-p) / p}$ for $i \notin \Lambda$; then $\operatorname{det} A=1$.

If $B$ is not diagonal we simply diagonalize $B$. Let $T$ be the matrix that diagonalizes $B$, i.e. $B=T^{*} D_{B} T$, where $D_{B}$ has the eigenvalues of $B$ on the 
diagonal. Now

$$
\begin{aligned}
\operatorname{det} B & =\operatorname{det} D_{B}=\left(\frac{1}{n} \inf _{A \in \mathcal{A}}\left\{\operatorname{tr}\left(A D_{B}\right)\right\}\right)^{n}=\left(\frac{1}{n} \inf _{A \in \mathcal{A}}\left\{\operatorname{tr}\left(A T^{*} B T\right)\right\}\right)^{n} \\
& =\left(\frac{1}{n} \inf _{A \in \mathcal{A}}\left\{\operatorname{tr}\left(T A T^{*} B\right)\right\}\right)^{n}=\left(\frac{1}{n} \inf _{A^{\prime} \in \mathcal{A}}\left\{\operatorname{tr}\left(A^{\prime} B\right)\right\}\right)^{n} .
\end{aligned}
$$

The last equality is just change of coordinates.

REMARK. The proof of Lemma 1.1 gives an algorithm for calculating the matrix $A$ :

(1) Suppose $B$ is a Hermitian matrix. Calculate the diagonalization matrix $T$ such that $T^{*} B T=\operatorname{diag}\left(\lambda_{1}, \ldots, \lambda_{n}\right)$, where $\lambda_{k} \leq \lambda_{k+1}$.

(2) (a) If $\operatorname{det} B \neq 0$ let $A^{\prime}=(\operatorname{det} B)^{1 / n} \operatorname{diag}\left(1 / \lambda_{1}, \ldots, 1 / \lambda_{n}\right)$.

(b) If $\operatorname{det} B=0$, and $B$ has rank $n-p$, let

$$
A_{k}^{\prime}=k^{n-p} \operatorname{diag}(k, \ldots, k, 1 / k, \ldots, 1 / k),
$$

with $p k^{\prime}$ s.

(3) (a) If $\operatorname{det} B \neq 0$, let $A=T A T^{*}$, and we have

$$
\operatorname{det} B=\left(\frac{1}{n} \operatorname{tr}(A B)\right)^{n} \text {. }
$$

(b) If $\operatorname{det} B=0$, let $A_{k}=T A_{k} T^{*}$, and we have

$$
\operatorname{det} B=\lim _{k \rightarrow \infty}\left(\frac{1}{n} \operatorname{tr}\left(A_{k} B\right)\right)^{n} \text {. }
$$

The algorithm above must of course be improved if used in a numerical calculation.

2. Some applications of Bellman's principle to the complex Monge-Ampère operator. The complex Monge-Ampère operator, $\left(\mathrm{dd}^{\mathrm{c}} u\right)^{n}$, can be defined on quite general sets of plurisubharmonic functions (see e.g. [BT76], [Ceg98]). If $u \in \mathcal{C}^{2}(\Omega)$ then

$$
\left(\operatorname{dd}^{\mathrm{c}} u\right)^{n}=4^{n} \operatorname{det}\left(\frac{\partial^{2} u}{\partial z_{j} \partial \bar{z}_{k}}\right) d V,
$$

where $d V$ is the volume measure in $\mathbb{C}^{n}$. Let us denote the above determinant by $M(u)$.

If $u$ is a $\mathcal{C}^{1,1}$ function, then $u$ is twice differentiable almost everywhere, and the second partial derivatives of $u$ defined pointwise as locally bounded functions coincide with distributional derivatives. Furthermore (2.1) holds for $u \in \mathcal{C}^{1,1}$ (see [Bło96]). 
For every complex $n \times n$ Hermitian positive matrix $A$, consider the Kähler form

$$
\Delta_{A}=\frac{1}{n} \sum_{j, k} \frac{a_{i j} \partial^{2}}{\partial z_{j} \partial \bar{z}_{k}} .
$$

Then

$$
M(u(z))=\left(\inf _{A \in \mathcal{A}} \Delta_{A} u(z)\right)^{n} \quad \text { if } u \in \mathcal{P} \mathcal{S H} \cap \mathcal{C}^{1,1} .
$$

As it is written, (2.3) does not hold on less smooth plurisubharmonic functions since the right hand side is a distribution, and it is generally hard to multiply distributions.

\subsection{Examples}

EXAmPle 2.1. Let $u(z)=\left|z_{1} z_{2}\right|^{2}$. Then the Hessian $H_{u}$ is given by

$$
H_{u}(z)=\left[\begin{array}{ll}
\left|z_{1}\right|^{2} & z_{1} \bar{z}_{2} \\
z_{2} \bar{z}_{1} & \left|z_{2}\right|^{2}
\end{array}\right],
$$

and the Monge-Ampère operator of $u$ is zero everywhere.

$H_{u}$ has eigenvalues $\lambda_{1}=0$ and $\lambda_{2}=\left|z_{1}\right|^{2}+\left|z_{2}\right|^{2}$, and away from both coordinate axes, is diagonalized by

$$
T_{u}=\left[\begin{array}{cc}
-\frac{\bar{z}_{2}}{\bar{z}_{1} \sqrt{1+\left|z_{2} / z_{1}\right|^{2}}} & \frac{1}{\sqrt{1+\left|z_{2} / z_{1}\right|^{2}}} \\
\frac{z_{1}}{z_{2} \sqrt{1+\left|z_{1} / z_{2}\right|^{2}}} & \frac{1}{\sqrt{1+\left|z_{1} / z_{2}\right|^{2}}}
\end{array}\right] .
$$

Since

$$
D=T_{u} H_{u} T_{u}^{*}=\left[\begin{array}{cc}
0 & 0 \\
0 & \left|z_{1}\right|^{2}+\left|z_{2}\right|^{2}
\end{array}\right]
$$

we choose, according to the proof of Lemma 1.1,

$$
A=\left[\begin{array}{cc}
k & 0 \\
0 & 1 / k
\end{array}\right] \text {. }
$$

Straightforward calculations give

$$
\operatorname{tr}\left(T_{u}^{*} A T_{u} H_{u}\right)=\frac{\left|z_{1}\right|^{2}+\left|z_{2}\right|^{2}}{k} .
$$

The Monge-Ampère operator of $u$ is zero and there is an eigenvalue $\lambda_{1}=0$ everywhere. Our choice of $T_{u}$ is a change of coordinates in $\mathbb{C}^{2}$ such that in the new coordinate $z^{\prime}=T_{u}(z)$ we have $\partial_{z_{1}^{\prime} \bar{z}_{1}^{\prime}}^{2} u=0$. That is, $u$ is harmonic on the line $\left\{z_{2}^{\prime}=0\right\}$. 
EXAmple 2.2. Let $u=\left|z_{1}+z_{3}\right|^{4}+\left|z_{2}\right|^{4}$. Then the Hessian $H_{u}$ is given by

$$
H_{u}(z)=\left(\begin{array}{ccc}
4\left|z_{1}+z_{3}\right|^{2} & 0 & 4\left|z_{1}+z_{3}\right|^{2} \\
0 & 4\left|z_{2}\right|^{2} & 0 \\
4\left|z_{1}+z_{3}\right|^{2} & 0 & 4\left|z_{1}+z_{3}\right|^{2}
\end{array}\right),
$$

and the Monge-Ampère operator of $u$ is zero everywhere. Suppose we stay away from $z_{2}=0$. Then $H_{u}$ has eigenvalues $\lambda_{1}=0, \lambda_{2}=4\left|z_{2}\right|^{2}$, and $\lambda_{3}=8\left|z_{1}+z_{3}\right|^{2}$, and is diagonalized by

$$
T_{u}=\left(\begin{array}{ccc}
-1 / 2 & 0 & 1 / 2 \\
0 & 1 & 0 \\
1 / 2 & 0 & 1 / 2
\end{array}\right)
$$

We choose, according to the proof of Lemma 1.1,

$$
A=\operatorname{diag}\left(k^{2}, 1 / k, 1 / k\right) .
$$

Straightforward calculations give

$$
\operatorname{tr}\left(T_{u}^{*} A T_{u} H_{u}\right)=\frac{4\left|z_{2}\right|^{2}+4\left|z_{1}+z_{3}\right|^{2}}{k} .
$$

If $z_{2}=0$ we get the eigenvalues $\lambda_{1,2}=0$ and $\lambda_{3}=8\left|z_{1}+z_{3}\right|^{2}$, and $H_{u}$ is diagonalized by exactly the same matrix $T_{u}$ as above. Choosing $A=$ $\operatorname{diag}\left(k, k, 1 / k^{2}\right)$, we get

$$
\operatorname{tr}\left(T_{u}^{*} A T_{u} H_{u}\right)=\frac{8\left|z_{1}+z_{3}\right|^{2}}{k}
$$

If $z_{3}+z_{1}=0$, we get the eigenvalues 0 and $4\left|z_{2}\right|^{2}$, and

$$
\operatorname{tr}\left(T_{u}^{*} A T_{u} H_{u}\right)=\frac{4\left|z_{2}\right|^{2}}{k^{2}} .
$$

In my opinion the examples above show quite clearly why the following theorem is true:

TheOREM 2.3 ([BK77]). Let $p \in \mathbb{C}^{2}$ and assume $u \in \mathcal{P S H} \cap \mathcal{C}^{3}$, and $\left(\mathrm{dd}^{\mathrm{c}} u\right)^{2}=0$ in a neighbourhood of $p$. If $\mathrm{dd}^{\mathrm{c}} u(p) \neq 0$, then there is a complex manifold $M$ through $p$ such that $\left.u\right|_{M}$ is harmonic on $M$.

Proof. Let $H_{u}$ be the complex Hessian of $u$. The null space of $H_{u}$ is a complex subspace of $\mathbb{C}^{2}$. Thus the integral curves of the vector field of eigenvectors corresponding to the eigenvalue 0 over every point form the required complex manifold $M$. Since $0=M_{u}=\lim _{k \rightarrow 0} \operatorname{tr}\left(A_{k}^{\prime} H_{u}\right)$, where $A_{k}^{\prime}=T_{u}^{*} \operatorname{diag}(k, 1 / k) T_{u}$, and $T_{u}$ is the matrix that diagonalizes $H_{u}$, it is clear that $u$ is harmonic along $M$. 
Using estimates for the energy class $\mathcal{F}$ of plurisubharmonic functions with bounded (classical) energy, developed in the papers [Ceg98], [Ceg01], and [Ceg02] we can generalize two theorems by Błocki [Bło96].

TheOREm 2.4. Let $\Omega$ be a hyperconvex set and suppose $u \in \mathcal{F}(\Omega)$. Furthermore assume that $\nu$ is a positive measure absolutely continuous with respect to the Lebesgue measure on $\Omega$. Then the following are equivalent:

(1) $\left(\mathrm{dd}^{\mathrm{c}} u\right)^{n} \geq \nu$,

(2) $\Delta_{A} u \geq \nu^{1 / n}$ for all $A \in \mathcal{A}$,

(3) $\left(\mathrm{dd}^{\mathrm{c}} u_{\delta}\right)^{n} \geq\left(\nu^{1 / n} * \varrho_{\delta}\right)^{n}$, where $u=\varrho_{\delta}$ is the usual regularization with an approximative identity.

Proof. Part of this proof is just a line by line copy of the proof of Theorem 3.10 in [Bło96].

We begin by showing that $(3) \Rightarrow(1)$. Since $\left(\mathrm{dd}^{\mathrm{c}} u_{\delta}\right)^{n} \rightarrow\left(\mathrm{dd}^{\mathrm{c}} u\right)^{n}$ weakly as $\delta \rightarrow 0$, the implication follows from the convergence of $\nu^{1 / n} * \varrho_{\delta}$.

Assume $\Delta_{A} u \geq \nu^{1 / n}$ for all $A \in \mathcal{A}$. From (2.3) it follows that $\left(\mathrm{dd}^{\mathrm{c}} u_{\delta}\right)^{n}=$ $\left(\inf \Delta_{A} u_{\delta}\right)^{n}=\left(\inf \left(\Delta_{A} u\right) * \varrho_{\delta}\right)^{n} \geq \nu^{1 / n}$. Note that since $u \in \mathcal{F}, u$ has finite classical energy so the second to last equality holds. Hence $(2) \Rightarrow(3)$.

To show that $(1) \Rightarrow(2)$, fix $A \in \mathcal{A}$ and take any plurisubharmonic $\psi \in$ $\varepsilon_{0} \cap \mathcal{C}^{\infty}$. Set $G:=\left(\mathrm{dd}^{\mathrm{c}} \psi\right)^{n}$. Then $G^{1 / n}$ is smooth. Let $B$ be any ball in $\Omega$ and solve the equation $\Delta_{A} \varphi=G^{1 / n}$ with boundary data $\varphi=\psi$ on $\partial B$. It is clear that $\varphi \in \mathcal{C}^{\infty}(B)$, and from (2.3) it follows that $\left(\operatorname{dd}^{\mathrm{c}} \varphi\right)^{n} \leq G$ wherever $\varphi$ is plurisubharmonic. According to [Bło96, Theorem 3.9, p. 735] it follows that $\psi \leq \varphi$, and so $\int_{B} \Delta_{A} \psi \geq \int_{B} \Delta_{A} \varphi \geq \int_{B} G^{1 / n} d V$.

Let $u \in \mathcal{F}(\Omega)$. Then there is a sequence $\left\{\psi_{j}\right\}_{j} \subset \mathcal{E}_{0} \cap \mathcal{C}^{\infty}(\Omega)$ such that $\psi_{j} \rightarrow u$ in capacity $($ see $[\operatorname{Ceg} 02])$. Let $G_{j}=\left(\operatorname{dd}^{\mathrm{c}} \psi_{j}\right)^{n}$. Then $\Delta_{A} \psi_{j} \geq G_{j}^{1 / n}$ and since $\left(\mathrm{dd}^{\mathrm{c}} \psi_{j}\right)^{n}$ converges weak ${ }^{*}$ to $\left(\mathrm{dd}^{\mathrm{c}} u\right)^{n}$ as $j \rightarrow \infty$, we get $\Delta_{A} u$ $\geq \nu^{n}$.

Using the fact that the mapping $(\operatorname{det} B)^{1 / n}$ is concave on the set of positive definite Hermitian matrices, together with Theorem 2.4 one gets the following subadditivity theorem:

THEOREm 2.5. Let $\Omega$ be a hyperconvex set and suppose $u, v \in \mathcal{F}(\Omega)$. Furthermore assume that $\nu$ and $\mu$ are positive measures absolutely continuous with respect to the Lebesgue measure on $\Omega$, and that $\left(\mathrm{dd}^{\mathrm{c}} u\right)^{n} \geq \mu$ and $\left(\operatorname{dd}^{\mathrm{c}} v\right)^{n} \geq \nu$. Then

$$
\left(\operatorname{dd}^{\mathrm{c}}(u+v)\right)^{n} \geq\left(\mu^{1 / n}+\nu^{1 / n}\right)^{n} .
$$

Note how this contrasts with the following lemma: 
Lemma 2.6. Suppose $\Omega$ is a hyperconvex domain in $\mathbb{C}^{n}$ and that $u, v \in$ $\mathcal{F}(\Omega)$. Then

$$
\int_{\Omega}\left(\operatorname{dd}^{\mathrm{c}}(u+v)\right)^{n} \leq\left[\left(\int_{\Omega}\left(\mathrm{dd}^{\mathrm{c}} u\right)^{n}\right)^{1 / n}+\left(\int_{\Omega}\left(\mathrm{dd}^{\mathrm{c}} v\right)^{n}\right)^{1 / n}\right]^{n} .
$$

For a proof see [CW03].

3. Lelong numbers. The Lelong number of a function $u$ at $x \in \mathbb{C}^{n}$ is defined as

$$
\nu(u, x)=\lim _{r \rightarrow 0} \frac{1}{(2 \pi)^{n}} \int_{\|z-x\| \leq r} \mathrm{dd}^{\mathrm{c}} u \wedge\left(\mathrm{dd}^{\mathrm{c}} \log \|z-x\|\right)^{n-1} .
$$

Let $\omega_{n}$ be the $(2 n-1)$-volume of the unit sphere in $\mathbb{C}^{n}$. Let $B(r ; x)$ be the ball of radius $r$ with center $x$, and write $\partial B(r ; x)=S(r ; x)$. Furthermore let $B(r)=B_{r}=B(r ; 0)$, and the same for the sphere. If we define

$$
\begin{aligned}
M(u, r, x) & =\sup _{z \in B(r ; x)} u(z), \\
\lambda(u, r, x) & =\frac{1}{\omega_{n}} \int_{S(1 ; 0)} u(x+r z) d S_{1}(z),
\end{aligned}
$$

we have

$$
\nu(u, x)=\lim _{r \rightarrow 0} \frac{M(u, r, x)}{\log r}=\lim _{r \rightarrow 0} \frac{\lambda(u, r, x)}{\log r}
$$

(see e.g. [Kis79]).

Given a function $u: \mathbb{C}^{n} \supset \Omega \rightarrow \mathbb{R} \cup\{-\infty\}$ we define the slice of $u$ through 0 and $p \in \mathbb{C}^{n}$ by $u_{p}(\zeta):=u(\zeta p), \zeta \in \mathbb{C}$, wherever this expression makes sense.

Lemma 3.1. If $u: \mathbb{C}^{n} \supset \Omega \rightarrow \mathbb{R} \cup\{-\infty\}$ and $0 \in \Omega$, then $\nu\left(u_{p}, 0\right) \geq$ $\nu(u, 0)$.

Proof. Since $\log r<0$ if $r<1$, we have

$$
\lim _{r \rightarrow 0} \frac{\sup _{\|z\| \leq r} u(z)}{\log r} \leq \lim _{r \rightarrow 0} \frac{\sup _{\|\zeta\| \leq r} u(\zeta p)}{\log r} .
$$

Using this lemma we can prove that the reverse inequality holds almost everywhere.

Lemma 3.2. If $u \in \mathcal{P S H}(B)$, then $\nu\left(u_{q}, 0\right)=\nu(u, 0)$ for all $q \in B \backslash A$, where $A$ is a set of Lebesgue measure zero.

Proof. This follows from

$$
\nu(u, 0)=\frac{1}{\omega_{n}} \int_{S(1)} \nu(u(\zeta y), 0) d S_{1}(y),
$$

and from Lemma 3.1 above. 
For functions radial in at least one variable Lemma 3.2 above can be considerably strengthened.

Lemma 3.3. Assume $u: \mathbb{C}^{2} \supset B \rightarrow \mathbb{R} \cup\{-\infty\}, u \in \mathcal{P S H}(B)$, and $u(|z|, w)=u(z, w)$. Suppose that $\nu(u, 0)=0$. Then for all $y=\left(y_{1}, y_{2}\right) \in B$ such that $y_{1} y_{2} \neq 0, \nu\left(u_{y}, 0\right)=0$.

Proof. Take $p=(z, w), q=\left(z^{\prime}, w^{\prime}\right) \in \mathbb{C}^{2}$ with $|z|<\left|z^{\prime}\right|$ and $|w|=\left|w^{\prime}\right|=R$. Since $u(r, w)$ is an increasing function in the radial variable we have

$$
\sup _{|w|=R} u(|z|, w) \leq \sup _{\left|w^{\prime}\right|=R} u\left(\left|z^{\prime}\right|, w^{\prime}\right) .
$$

That is, $\nu\left(u_{p}, 0\right) \geq c \nu\left(u_{q}, 0\right)$ for some constant $c$. Take $y=\left(y_{1}, y_{2}\right)$ such that neither $y_{1}$ nor $y_{2}$ is zero. According to Lemma 3.2 there is a point $y^{\prime}=\left(y_{1}^{\prime}, y_{2}\right)$ with $\left|y_{1}^{\prime}\right|<\left|y_{1}\right|$ such that $\nu\left(u_{y^{\prime}}, 0\right)=0$, but then $0 \geq \nu\left(u_{y}, 0\right)$.

4. Bellman's principle for polyradial functions. In $\mathbb{C}^{n}$ it is well known that the Lelong number is dominated by the Monge-Ampère operator in the following way:

$$
(2 \pi \nu(u, x))^{n} \leq\left(\mathrm{dd}^{\mathrm{c}} u\right)^{n}(\{x\}) .
$$

Note that if $u\left(z_{1}, z_{2}\right)=\max \left\{1 / k \log \left|z_{1}\right|, k \log \left|z_{2}\right|\right\}$, then one can show that $\left(\operatorname{dd}^{\mathrm{c}} u\right)^{2}(0)=4 \pi^{2} \delta_{0}$, and $\nu(u, 0)=1 / k$, so inequality (4.1) cannot be reversed.

For polyradial functions at least, it seems that the "correct" version of (4.1) should be in the flavor of Bellman's principle.

We begin with a simple lemma, concerning subharmonic functions in the complex plane.

Lemma 4.1. Let $D$ denote the unit disc in $\mathbb{C}^{1}$, and suppose $u \in \mathcal{S H}(D)$, $u \not \equiv-\infty$, is radial (i.e. $u(|z|)=u(z))$. Take $\varepsilon>0$, and let $D_{\varepsilon}=\{z: u(z)>$ $(k+\varepsilon) \log |z|\} \cup\{0\}$. Then $\nu(u, 0)=k=\mathrm{const} \cdot \partial \bar{\partial} u(\{0\})$ if and only if $D_{\varepsilon}$ is a disc of positive radius centered at the origin.

Proof. Since $u$ is radial and $u \not \equiv-\infty$, it is a convex function in $\log r$, continuous except for a possible pole at the origin. The result follows.

The following theorem, for polyradial functions, is an analogue to Bellman's principle for plurisubharmonic twice differentiable functions.

Theorem 4.2. Let $\Omega$ be a domain in $\mathbb{C}^{n}$, containing the origin. Assume $u: \mathbb{C}^{n} \rightarrow \mathbb{R}$ is plurisubharmonic and polyradial on $\Omega$. Take any point $p_{j}$ on the $z_{j}$-axis, and set

$$
v_{j}=\nu_{p_{j}}(u, 0) .
$$

Then $\left(\mathrm{dd}^{\mathrm{c}} u\right)^{n} \leq(2 \pi)^{n} v_{1} \cdots v_{n}$. 
Proof. For simplicity, assume that the unit polydisc $D_{1} \times \cdots \times D_{1}$ is contained in $\Omega$.

Fix $\varepsilon>0$. According to Lemma 4.1 there is a disc $D_{\varepsilon}^{1} \subset \mathbb{C}^{1}$ such that $u(z) \geq\left(v_{1}+\varepsilon\right) \log \left|z_{1}\right|$ on $D_{\varepsilon}^{1} \times\{0\} \times \cdots \times\{0\}$. Using the maximum principle in one complex variable, and the fact that $u$ is polyradial, we clearly obtain $u(z) \geq\left(v_{1}+\varepsilon\right) \log \left|z_{1}\right|$ on $D_{\varepsilon}^{1} \times D_{1} \times \cdots \times D_{1}$. As a result, $\max \left\{u(z),\left(v_{1}+\varepsilon\right) \log \left|z_{1}\right|\right\}=u(z)$ on $D_{\varepsilon}^{1} \times D_{1} \times \cdots \times D_{1}$.

By repeating this reasoning one gets

$$
\max \left\{u(z),\left(v_{1}+\varepsilon\right) \log \left|z_{1}\right|, \ldots,\left(v_{n}+\varepsilon\right) \log \left|z_{n}\right|\right\}=u(z)
$$

on $D_{\varepsilon}^{1} \times \cdots \times D_{\varepsilon}^{1}$. On the other hand

$$
\begin{aligned}
\max \left\{u(z),\left(v_{1}+\varepsilon\right) \log \left|z_{1}\right|, \ldots,\left(v_{n}+\varepsilon\right) \log \left|z_{n}\right|\right\} & \\
\geq \max & \left\{\left(v_{1}+\varepsilon\right) \log \left|z_{1}\right|, \ldots,\left(v_{n}+\varepsilon\right) \log \left|z_{n}\right|\right\} .
\end{aligned}
$$

Let $u_{\varepsilon}(z)=\max \left\{u(z),\left(v_{1}+\varepsilon\right) \log \left|z_{1}\right|, \ldots,\left(v_{n}+\varepsilon\right) \log \left|z_{n}\right|\right\}$ for $\varepsilon>0$. Then $u_{\varepsilon}=u$ on $D_{\varepsilon}^{1} \times \cdots \times D_{\varepsilon}^{1}$, hence

$$
\int_{D_{\varepsilon}^{1} \times \cdots \times D_{\varepsilon}^{1}}\left(\mathrm{dd}^{\mathrm{c}} u\right)^{n}=\int_{D_{\varepsilon}^{1} \times \cdots \times D_{\varepsilon}^{1}}\left(\mathrm{dd}^{\mathrm{c}} u_{\varepsilon}\right)^{n} \leq \int_{D_{1} \times \cdots \times D_{1}}\left(\operatorname{dd}^{\mathrm{c}} u_{\varepsilon}\right)^{n} .
$$

By the approximation theorem in [Ceg01] and monotone convergence, for every $\varepsilon>0$ we have $\left(\operatorname{dd}^{\mathrm{c}} u_{\varepsilon}\right)^{n} \rightarrow(2 \pi)^{n} v_{1} \cdots v_{n}$ as $\varepsilon \rightarrow 0$.

To sum up, given a polyradial plurisubharmonic function $u$, we have

$$
(2 \pi \nu(u, 0))^{n} \leq\left(\operatorname{dd}^{\mathrm{c}} u\right)^{n}(\{0\}) \leq(2 \pi)^{n} v_{1} \cdots v_{n},
$$

where $v_{k}$ is the Lelong number along the $k$ th axis.

(4.2) is perhaps not so surprising if we consider the following special case of the Hadamard inequality:

Proposition 4.3. Let $B=\left(b_{i j}\right)$ be a positive semidefinite Hermitian matrix. Then $\operatorname{det} B \leq \prod b_{i i}$.

Proof. Since we might as well assume that $\operatorname{det} B \neq 0$, all diagonal elements $b_{i i}$ are nonzero. Let $D=\operatorname{diag}\left(b_{11}^{-1 / 2}, \ldots, b_{n n}^{-1 / 2}\right)$. Since $(D B D x, x)=$ $(B D x, D x)$, we see that $D B D$ is positive semidefinite and thus

$$
\frac{\operatorname{det} B}{\prod b_{i i}}=\operatorname{det}\left(B D^{2}\right)=\operatorname{det}(D B D) \leq\left(\frac{1}{n} \operatorname{tr}(D B D)\right)^{n}=1
$$

where the inequality follows, as in the proof of Lemma 1.1, by comparison between arithmetic and geometric means.

Note that Proposition 4.3 above is a nice analogue to Theorem 4.2 if we interpret $\operatorname{det} B$ as the residual Monge-Ampère mass at the origin of a polyradial function $u$, and $b_{i i}$ as $\nu\left(u_{i}, 0\right)$, where $u_{1}: \mathbb{C} \rightarrow \mathbb{R} \cup\{-\infty\}$ is the slice of $u$ along the $z_{1}$-axis, defined by $u_{1}(\zeta)=u(\zeta, 0, \ldots, 0)$ and so forth. 
It is well known that for a large class of functions $u$, the determinant of the Hessian, $\operatorname{det} \partial_{j \bar{k}}^{2} u$, can be generalized to a measure. However it is not at all clear to me how to generalize $\prod \partial_{k \bar{k}}^{2} u$ for plurisubharmonic functions from some reasonable class.

We finish with an application of Proposition 4.3 to a very simple case. Let $u=\max \left(a_{1} \log \left|z_{1}\right|, \ldots, a_{n} \log \left|z_{n}\right|\right)$, and set $u_{m}=\max (u,-m)$. Let $u_{m}^{j}$ be a family of smooth plurisubharmonic functions, decreasing to $u_{m}$. Then $\left(\mathrm{dd}^{\mathrm{c}} u_{m}^{j}\right)^{n} \rightarrow\left(\mathrm{dd}^{\mathrm{c}} u_{m}\right)^{n}$. Furthermore

$$
\int M\left(u_{m}^{j}\right) \leq \int \prod \partial_{k \bar{k}}^{2} u_{m}^{j}
$$

by Proposition 4.3. Note that, in measure, $\partial_{1 \overline{1}}^{2} u_{m}^{j} \rightarrow 2 \pi a_{1} \lambda_{1}$, where $\lambda_{1}$ is Lebesgue measure on the segment $\left|z_{1}\right|=e^{-m / a_{1}}$, and $\left|z_{k}\right| \leq e^{-m / a_{k}}$. Thus it follows from (4.3) that

$$
\int\left(\operatorname{dd}^{\mathrm{c}} u_{m}\right)^{n} \leq(2 \pi)^{n} \prod a_{k},
$$

and using Bellman's principle on the sequence $u_{m}^{j}$ above we obtain an inequality in the other direction. Hence $\left(\operatorname{dd}^{\mathrm{c}} u\right)^{n}\{0\}=(2 \pi)^{n} \prod a_{k}$. Note that with the notation of Theorem $4.2, \nu_{k}=a_{k}$.

\section{References}

[BK77] E. Bedford and M. Kalka, Foliations and complex Monge-Ampère equations, Comm. Pure Appl. Math. 30 (1977), 543-571.

[Bło96] Z. Błocki, The complex Monge-Ampère operator in hyperconvex domains, Ann. Scuola Norm. Sup. Pisa Cl. Sci. (4) 23 (1997), 721-747.

[BT76] E. Bedford and B. A. Taylor, The Dirichlet problem for a complex MongeAmpère equation, Invent. Math. 37 (1976), 1-44.

[Ceg98] U. Cegrell, Pluricomplex energy, Acta Math. 180 (1998), 187-217.

[Ceg01] -, Convergence in capacity, technical report NI01046-NPD, Isaac Newton Inst. for Math. Sci., Cambridge, 2001.

[Ceg02] —, Exhaustion functions for hyperconvex domains, manuscript, 2002.

[CW03] U. Cegrell and J. Wiklund, Monge-Ampère norm for delta-plurisubharmonic functions, manuscript, 2003.

[Gav77] B. Gaveau, Méthodes de contrôle optimal en analyse complexe. I. Résolution d'équations de Monge-Ampère, J. Funct. Anal. 25 (1977), 391-411.

[Kis79] C. O. Kiselman, Densité des fonctions plurisousharmoniques, Bull. Soc. Math. France 107 (1979), 295-304.

[MM64] M. Marcus and H. Minc, A Survey of Matrix Theory and Matrix Inequalities, Allyn and Bacon, 1964.

Matematiska Institutionen

Umeå Universitet

S-901 87 Umeå, Sweden

E-mail: jonas.wiklund@math.umu.se 